\title{
Sliding modes in a class of complex-valued nonlinear systems
}

\author{
Arnau Dòria-Cerezo, Josep M. Olm, Domingo Biel, and Enric Fossas
}

\begin{abstract}
A number of physical systems allow a complexvalued representation. This paper extends the theory of sliding modes to a class of nonlinear systems described by complexvalued variables. Hence, states, parameters, control actions, and sliding manifolds belong, in general, to the complex field. It is also shown in the article that the proposed design in the complex-valued framework provides shorter reaching times to the sliding manifold than the standard sliding mode design at equal initial condition and control effort. Different implementation approaches are also evaluated, and numerical examples illustrate the proposal.
\end{abstract}

Index Terms-Complex valued dynamical systems, sliding modes

\section{INTRODUCTION}

$\mathbf{T}$ HE representation of physical systems using a ComplexValued State-Space (CVSS) is not new. Many examples refer to three-phase electrical systems [1], where this description allows order reduction and simplifies the analysis. Examples in the electrical systems field include electrical machines [2], and power converters [3] [4], while other applications deal with mechanical rotating systems [5], the study of mechanical vibrations [6], complex-valued neural networks [7], band-pass filters $[8] \ldots$

Control theory tools for complex-valued systems are not extensive and include, for example, the generalisation of the Routh-Hurwitz test for complex polynomials, proposed in [9] and extended in [10], [11]. More recently, complex extensions of other linear control techniques such as the root locus method [12] and frequency-domain analysis [13] have also been reported. However, most of the referenced techniques are oriented to the linear case, and nonlinear complex-valued systems are less studied. Among the few examples found are stability analysis of complex-valued differential equations and delay differential equations, which are discussed in [14] and [15], respectively.

In this paper, sliding modes are extended to a class of complex-valued nonlinear dynamical systems. Sliding mode control using complex-valued manifolds was used in [16] to

The work was partially supported by the Spanish Government through the Agencia Estatal de Investigación Project DPI2017-85404-P and by the Catalan Government through the Project 2017SGR872.

A. Dòria-Cerezo is with the Dept. of Electrical Engineering and the Inst. of Industrial and Control Engineering, Universitat Politècnica de Catalunya, Spain. e-mail: (arnau.doria@upc.edu).

J.M. Olm is with the Dept. of Mathematics and the Inst. of Industrial and Control Engineering, Universitat Politècnica de Catalunya, Spain.

D. Biel is with the Dept. of Electronics and the Inst. of Industrial and Control Engineering, Universitat Politècnica de Catalunya, Spain.

E. Fossas is with the Dept. of Automatic Control and the Inst. of Industrial and Control Engineering, Universitat Politècnica de Catalunya, Spain. seek for higher control flexibility using a complexification of a real-valued dynamics. Differently from [16], that was limited to linear systems and where the complexification was used to generalise the control design, the starting point of this paper are nonlinear systems with complex-valued dynamics.

The resulting complex-valued sliding modes can be seen as induced by a multidimensional variable structure controller. In particular, the proposal resembles the discontinuous state feedback controllers for real systems introduced in [17], [18], [19] -also known as unit control design methods [20]- which ensure sliding motion on a prescribed manifold. However, in this paper systems are described by complex-valued state space models, thus allowing its dynamic analysis and control to be carried out in a natural frame and with reduced order with respect to the corresponding real representation.

It is also shown in the paper that, at equal initial condition and control effort, the sliding manifold's reaching times achieved with the proposed complex-based controller are shorter than those obtained with a standard sliding mode control design. Such a result, which can be straightforwardly extended to the unit control methods, is not reported in the referenced papers, and underlines an additional benefit in using both this or the complex-valued control approach. Moreover, the implementation of complex sliding modes by means of some continuous and hysteretic approximations is discussed. Finally, a few examples are presented to illustrate the proposal, including a complex-based sliding observer designed to estimate the rotor flux of an induction motor.

The remainder of the paper is structured as follows. The basics of sliding mode control theory for compled-valued dynamical systems are set in Section II. Then, a comparative analysis of the reaching times achieved with complex-based and standard sliding modes is performed in Section III. The implementation of complex sliding mode controllers is discussed in IV. Numerical examples are presented in Section V. Finally, conclusions are drawn in Section VI.

Notation. $j=\sqrt{-1}$ is used, instead of $i$, to avoid confusion with electrical currents used in the application examples; $\mathbb{C}^{n}$ denotes the complex $n$ th-dimensional space; $\Omega \subset \mathbb{C}^{n}$ denotes an open subset of $\mathbb{C}^{n} ; H\left(\Omega, \mathbb{C}^{n}\right)$ denotes the set of holomorphic maps from $\Omega$ to $\mathbb{C}^{n} ; \mathcal{C}\left(\Omega, \mathbb{R}_{\geq 0}\right)$ denotes the set of continuous maps from $\Omega$ to $\mathbb{R}_{\geq 0} ; \operatorname{Re}(z)$ and $\operatorname{Im}(z)$ denote the real and imaginary parts, respectively, of $z \in \mathbb{C} ; z^{*}$ denotes the conjugate of $z \in \mathbb{C} ;|z|$ and $\varphi_{z}$ denote the magnitude and phase, respectively, of $z \in \mathbb{C}$; $\operatorname{sign}(z)$ denotes the sign function of a complex value $z \in \mathbb{C} \backslash\{0\}$, which is computed as in [21]:

$$
\operatorname{sign}(z)=\frac{z}{|z|} \text {. }
$$




\section{SLIDING MODE CONTROL OF CVSS SYSTEMS}

Consider a single input affine CVSS system as

$$
\dot{z}=f(z)+g(z) u,
$$

where $z \in \mathbb{C}^{n}$ is the state vector, $f, g \in H\left(\Omega, \mathbb{C}^{n}\right)$ are the system and control vectors, respectively, and

$$
u=u(z, \operatorname{sign}(\sigma)) \in \mathbb{C},
$$

is a switched control input signal, while $\sigma \in H(\Omega, \mathbb{C})$ is known as the complex switching function.

Definition 1 (Complex switching/sliding manifold \& sliding domain): Let system (2) be driven by the control law (3). The complex-valued set

$$
\mathcal{S}:=\{z \in \Omega ; \sigma(z)=0\}
$$

is called the complex switching manifold. If sliding motion is induced on $\Omega_{0} \subseteq \mathcal{S}$, then $\mathcal{S}$ is called the complex sliding manifold, and $\Omega_{0}$ is the sliding domain.

Definition 2 (Equivalent control): Let $\frac{\partial \sigma}{\partial z} g(z) \neq 0$, for all $z$ in a neighborhood of $\Omega_{0} \subseteq \mathcal{S}$. The equivalent control is the control law, $u=u_{e q}$, which makes $\mathcal{S}$ an invariant manifold with respect to (2). Namely,

$$
u_{e q}=-\left(\frac{\partial \sigma}{\partial z} g(z)\right)^{-1} \frac{\partial \sigma}{\partial z} f(z)
$$

Proposition 1: Let $\sigma \in H(\Omega, \mathbb{C})$ be a complex switching function, let $\mathcal{S}$ denote the corresponding switching manifold, and let also $k \in \mathbb{R}$. Assume that there exists $\Omega_{1} \subseteq \Omega$ with $\Omega_{1} \cap \mathcal{S} \neq \emptyset$, and $\epsilon_{1} \in \mathbb{R}^{+}$such that, for all $z \in \Omega_{1}$ :

$$
\left|\frac{\partial \sigma}{\partial z} g(z)\right| \geq \epsilon_{1} .
$$

Then, the switched control action

$$
u=u_{e q}+k e^{j\left(\pi-\varphi_{\sigma^{\prime} g}\right)} \operatorname{sign}(\sigma),
$$

with $u_{e q}$ defined in (4), induces sliding motion of system (2) on the sliding domain $\Omega_{1} \cap \mathcal{S}$. Moreover, for all $z(0)$ close enough to $\Omega_{1} \cap \mathcal{S}$, the sliding manifold is reached in finite time.

Proof: Consider the autonomous, positive definite auxiliary function

$$
V(z)=\frac{1}{2} \sigma^{*}(z) \sigma(z) .
$$

By assumption, $V \in \mathcal{C}\left(\mathbb{C}^{n}, \mathbb{R}_{\geq 0}\right)$ and it is locally Lipschitz in $z$. In turn, its time derivative over the trajectories of (2) for all $z \in \Omega_{1} \backslash \mathcal{S}$ is:

$$
\begin{aligned}
\dot{V} & =\frac{1}{2}\left(\sigma^{*} \dot{\sigma}+\sigma \dot{\sigma}^{*}\right)=\operatorname{Re}\left(\sigma^{*} \frac{\partial \sigma}{\partial z}(f(z)+g(z) u)\right)= \\
& =\operatorname{Re}\left(\sigma^{*} \frac{\partial \sigma}{\partial z} g(z)\left(u-u_{e q}\right)\right)=-\operatorname{Re}\left(k\left|\frac{\partial \sigma}{\partial z} g(z)\right||\sigma|\right)= \\
& =-k\left|\frac{\partial \sigma}{\partial z} g(z)\right||\sigma| .
\end{aligned}
$$

Now, using (5) it is immediate that

$$
\dot{V} \leq-k \epsilon_{1}|\sigma|
$$

and setting

$$
\gamma=\sqrt{2} k \epsilon_{1}>0
$$

(8) can be written as

$$
\dot{V} \leq-\gamma V^{\frac{1}{2}}
$$

Hence, $\dot{V}(z)<0$ for all $z$ in a neighborhood of $\Omega_{1} \backslash \mathcal{S}$, and (2) undergoes sliding motion on $\Omega_{1} \cap \mathcal{S}$ [20]. Moreover, it follows from (10) that $V(t)=0$ for $t \geq T$, with

$$
T=\frac{2}{\gamma} \sqrt{V(0)}
$$

this entailing the existence of $\bar{T} \in(0, T]$ such that

$$
\lim _{t \rightarrow \bar{T}} \sigma(z(t))=0
$$

therefore, $\mathcal{S}$ is reached in finite time for all $z(0)$ close enough to $\Omega_{1} \cap \mathcal{S}$.

Remark 1: The holomorfic character assumed for $f, g$, and $\sigma$ includes the case in which (2) is a linear system and $\mathcal{S}$ is a linear switching manifold, i.e.

$$
\begin{aligned}
& \dot{z}=A z+B u \\
& \sigma(z)=C z
\end{aligned}
$$

where $A$ is an $n \times n$ matrix with coefficients in $\mathbb{C}$, and $B, C \in$ $\mathbb{C}^{n}$.

Remark 2: Condition (5) is a technical assumption strongly related to the transversality condition, namely, $\frac{\partial \sigma}{\partial z} g(z) \neq 0$, already assumed in the definition of the equivalent control (recall Definition 2). Notice from (10), (9) that knowledge of $\epsilon_{1}$ allows to appropriately set the control gain, $k$, in case a specific reaching time is required.

Proposition 1 gives a control gain design criterion to meet the control goal that uses the equivalent control. However, when uncertainties are present it may be difficult to compute $u_{e q}$. An alternative for those cases in which an upper bound is known for $\left|u_{e q}\right|$ is given below.

Proposition 2: Let $\sigma \in H(\Omega, \mathbb{C})$ be a complex switching function, and let $\mathcal{S}$ denote the corresponding switching manifold. Assume that there exist $\Omega_{1} \subseteq \Omega$ with $\Omega_{1} \cap \mathcal{S} \neq \emptyset$, $\kappa \in \mathbb{C} \backslash\{0\}$, and $\epsilon_{1}, \epsilon_{2} \in \mathbb{R}^{+}$such that, for all $z \in \Omega_{1}$ :

$$
\begin{aligned}
& \left|\frac{\partial \sigma}{\partial z} g(z)\right| \geq \epsilon_{1} \\
& |\kappa| \cos \left(\varphi_{\sigma^{\prime} g}(z)+\varphi_{\kappa}\right)-\left|u_{e q}\right| \geq \epsilon_{2},
\end{aligned}
$$

with $u_{e q}$ defined in (4). Then, the switched control action

$$
u=-\kappa \operatorname{sign}(\sigma)
$$

induces sliding motion of system (2) on $\Omega_{1} \cap \mathcal{S}$. Moreover, for all $z(0)$ close enough to $\Omega_{1} \cap \mathcal{S}$, the sliding manifold is reached in finite time.

Proof: Consider again the autonomous, positive definite, Lipschitz continuous, auxiliary function defined in (7). Using 
(12), (4), its time derivative over the trajectories of (2) for all $z \in \Omega_{1} \backslash \mathcal{S}$ can be written as:

$$
\begin{aligned}
\dot{V}= & -\operatorname{Re}\left(\sigma^{*} \frac{\partial \sigma}{\partial z} g(z)\left(\kappa \operatorname{sign}(\sigma)+u_{e q}\right)\right)= \\
= & -|\sigma| \operatorname{Re}\left(\kappa \frac{\partial \sigma}{\partial z} g(z)+\frac{\partial \sigma}{\partial z} g(z) \frac{\sigma^{*}}{|\sigma|} u_{e q}\right)= \\
= & -|\sigma|\left|\frac{\partial \sigma}{\partial z} g(z)\right|\left(|\kappa| \cos \left(\varphi_{\sigma^{\prime} g}(z)+\varphi_{\kappa}\right)+\right. \\
& \left.+\left|u_{e q}\right| \cos \left(\varphi_{\sigma^{\prime} g}(z)+\varphi_{u_{e q}}(z)-\varphi_{\sigma}(z)\right)\right),
\end{aligned}
$$

where it has been taken into account that (1) immediately entails

$$
\operatorname{sign}(z)=e^{j \varphi_{z}}, \quad z \neq 0 .
$$

Now, using (11) it follows that

$$
\dot{V} \leq-\epsilon_{1}\left(|\kappa| \cos \left(\varphi_{\sigma^{\prime} g}(z)+\varphi_{\kappa}\right)-\left|u_{e q}\right|\right)|\sigma| \leq-\epsilon_{1} \epsilon_{2}|\sigma|,
$$

which can be written as (10) by setting

$$
\gamma=\sqrt{2} \epsilon_{1} \epsilon_{2}>0
$$

Hence, the result follows analogously as that in Proposition 1.

Remark 3: Condition (11a) is exactly the same as (5), so the first part of Remark 2 also applies to Proposition 2. As regards convergence time setting, notice from (10), (15), (11b) that, in this case, the adjustment is to be done not directly by the control gain but through the design parameter $\epsilon_{2}$, which is to be selected according to $\epsilon_{1}$. Once $\epsilon_{2}$ is fixed, the corresponding control gain, $\kappa$, is obtained from (11b) with $\left|u_{e q}\right|$ replaced by a known upper bound. Notice also that the closer the argument of $\kappa$ is to compensate that of $\sigma^{\prime} g$, the less control gain effort, $|\kappa|$, is required to fulfill (11b).

Remark 4: It is clear from (13) that the discontinuity in the control actions (6), (12) stems from the intrinsic singularity in the complex zero argument. Moreover, it is worth mentioning that analogous results for the real case under unit control are derived in [20]. In fact, (11b) is the complex-valued analogous of a condition on the control gain in classical unit control designs to guarantee finite-time convergence of sliding modes in systems with uncertainties in the open-loop vector field, $f$, while (11a) (or (5)) is also considered in unit control aiming at finite-time convergence [20].

\section{REACHING TIME REDUCTION}

The major flexibility in the control action selection given by complex sliding mode controllers with respect to the standard version may yield lower reaching times. This will be shown in this section for the control design introduced in Proposition 2 and linear switching functions, which can be represented as

$$
\sigma:=z_{1}
$$

without loss of generality. A similar computation, which is here omitted for the sake of brevity, can be carried out for the control design in Proposition 1.

A key feature of the proposed complex-valued sliding mode controller is the definition of the sign function as in (1), this immediately yielding (13). Hence, the control law (12) can be written as

$$
u=-\kappa e^{j \varphi_{\sigma}}, \quad \sigma \neq 0
$$

with $\kappa \in \mathbb{C} \backslash\{0\}$, which means that it can take any value on the circumference with radius $|\kappa|$. Instead, as indicated in the Introduction, complex-valued sliding modes can be treated as real-valued higher dimensional systems. In such a context, $\sigma \in$ $\mathbb{C}$ in (16) may be written as $(\operatorname{Re}(\sigma), \operatorname{Im}(\sigma))$, and its sign is to be computed componentwise. Hence, only four possibilities are allowed in the real case, namely,

$$
\operatorname{sign}(\sigma)=( \pm 1, \pm 1)=\sqrt{2}\left(\cos \varphi_{\sigma}, \sin \varphi_{\sigma}\right)
$$

with $\varphi_{\sigma}=\frac{(2 n+1) \pi}{4}, n=0,1,2,3$. Then, the standard sliding mode controller analogous for (12) may be written as

$$
u_{x}=-\kappa_{x} \operatorname{sign}(\sigma)=-\sqrt{2} \kappa_{x} e^{j \varphi_{\sigma}}, \quad \sigma \neq 0,
$$

where $\kappa_{x} \in \mathbb{C} \backslash\{0\}$ (in order to account for rotation-based decoupling terms in the control action), and it just takes four specific values on the circumference of radius $\sqrt{2}\left|\kappa_{x}\right|$. Therefore, a complex design with switching component (17) has a standard design analogous with switching component (18) and $\left|\kappa_{x}\right|=\frac{|\kappa|}{\sqrt{2}}$.

Now, recalling Remark 3 it will be assumed that

$$
\varphi_{\kappa}=\varphi_{\kappa_{x}}=-\varphi_{\sigma^{\prime} g}(z),
$$

this yielding restriction (11b) for a standard design to become

$$
\left|\kappa_{x}\right|-\left|u_{e q}\right|>\epsilon_{2} .
$$

Then, when following the proof of Proposition 2 for a standard design, relation (14) becomes

$$
\begin{aligned}
\dot{V} & \leq-\epsilon_{1}\left[\left|\kappa_{x}\right|(|\operatorname{Re}(\sigma)|+|\operatorname{Im}(\sigma)|)-\left|u_{e q}\right||\sigma|\right] \leq \\
& \leq-\epsilon_{1} \epsilon_{2}|\sigma|=-\gamma_{x} V^{\frac{1}{2}},
\end{aligned}
$$

an upper bound of the reaching time being

$$
T_{x}=\frac{2}{\gamma_{x}} \sqrt{V(0)},
$$

where $\gamma_{x}$ is the one in (15). Instead, for a complex design relation, using (20) we have that

$$
|\kappa|-\left|u_{e q}\right|=\sqrt{2}\left|\kappa_{x}\right|-\left|u_{e q}\right|>\sqrt{2} \epsilon_{2},
$$

and (14) becomes

$$
\dot{V} \leq-\sqrt{2} \epsilon_{2} \epsilon_{3}|\sigma|=-\sqrt{2} \gamma_{x} V^{\frac{1}{2}} .
$$

Therefore, an upper bound of the reaching time is

$$
T=\frac{T_{x}}{\sqrt{2}}<T_{x} .
$$

Remark 5: A straightforward extrapolation of this result allows to conclude that unit control designs also offer lower reaching times than standard sliding mode controllers. 


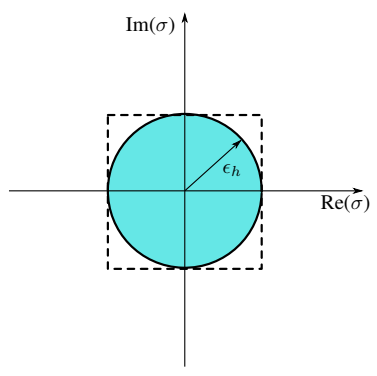

Fig. 1. Hysteresis area for the complex function. Filled area for Algorithms 1 and 3, and dotted area for the squared hysteresis in Algorithm 2.

\section{IMPLEMENTATION OF COMPLEX SLIDING CONTROLLERS}

Similarly to the standard sliding mode control approach, ideal complex sliding modes require infinite switching frequency, but this feature is unavailable in practical applications. In this section, different implementation strategies are proposed that approximate the complex sign function defined in (1), which appears in the switched control action proposed in (12), and the switching component in (6). Two approximations are considered: the boundary layer approach, and hysteresis functions.

\section{A. The boundary layer solution}

In standard sliding mode control theory, the boundary layer solution avoids discontinuities in the control action by approximating the sign function by a saturation term [22]. The boundary layer approach proposed here is equivalent to the one in [23], where a continuous unit control-type law is proposed for systems with bounded uncertainties. The complex-valued version is:

$$
u= \begin{cases}-\kappa \frac{\sigma}{|\sigma|} & \text { if }|\sigma|>\epsilon_{b} \\ -\kappa \frac{\sigma}{\epsilon_{b}} & \text { if }|\sigma| \leq \epsilon_{b}\end{cases}
$$

where $\epsilon_{b} \in \mathbb{R}^{+}$is the boundary layer width.

\section{B. Hysteretic approximations}

The hysteresis approximation for the complex sign function is illustrated in Figure 1. It basically defines a ball of radius $\epsilon_{h}$ in the complex plane representation of $\sigma$ where the output state is remembered. Out of this area, the complex function is evaluated. The hysteresis for the complex function (12) is implemented as shown in Algorithm 1, where $p$ refers to each iteration.

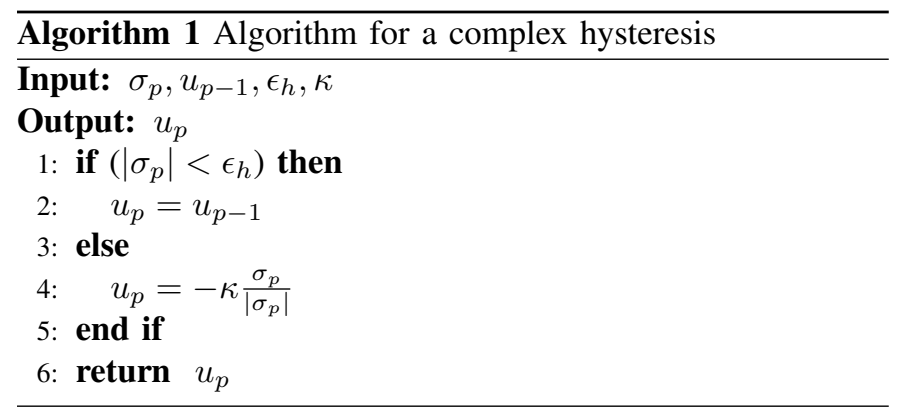

The area in the complex plane can be shaped depending on the switching dynamics, which will affect the chattering phenomena. See for example Algorithm 2, that implements the squared hysteresis area shown in Figure 1.

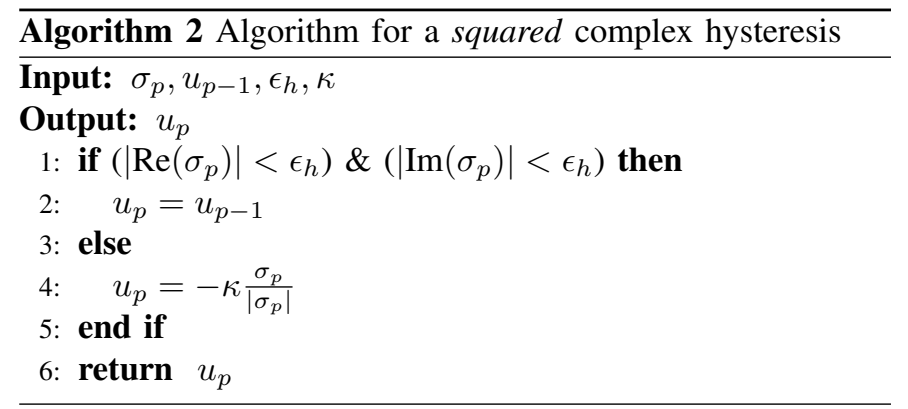

Standard multi-input sliding modes can be recovered if the control action takes discrete values, for example $\operatorname{Re}(u), \operatorname{Im}(u) \in\{-k, k\}$, where $k \in \mathbb{R}^{+}$. Then, Algorithm 1 can be modified as in Algorithm 3. More precisely, when the hysteresis is defined independently for the real and imaginary parts, the standard sliding mode control technique can be written as in Algorithm 4.
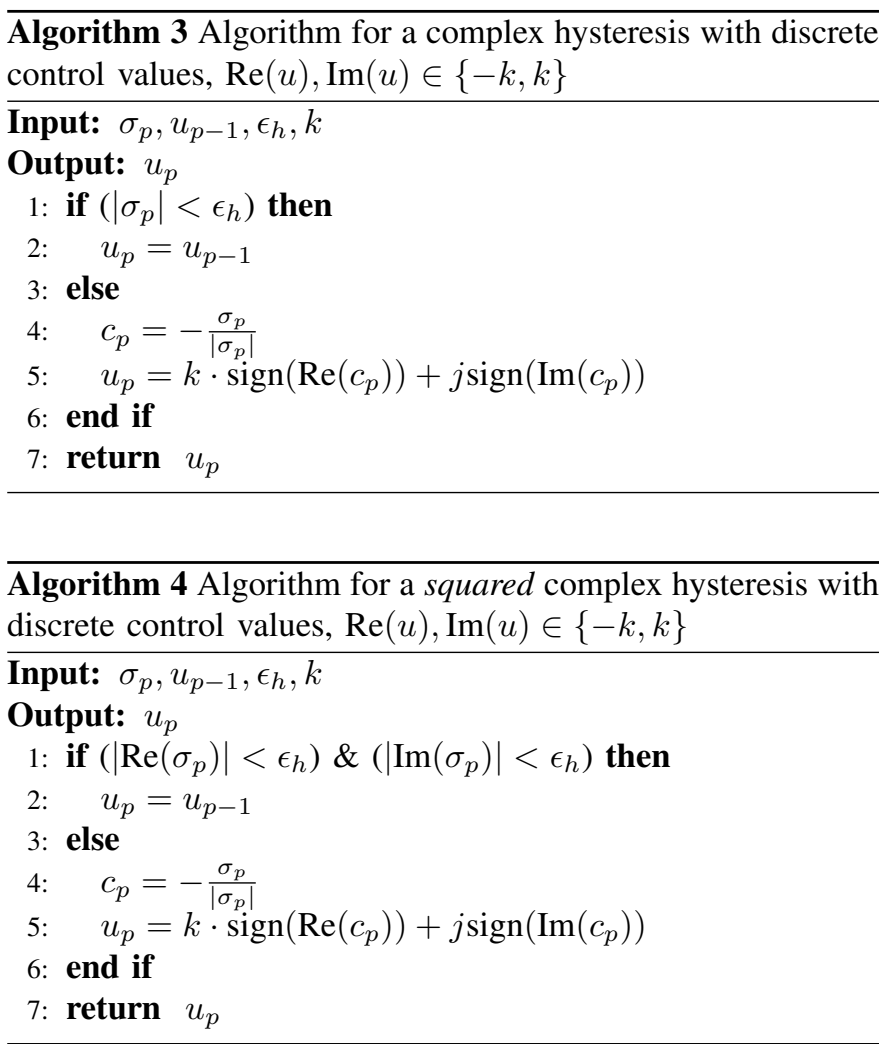

\section{EXAMPLES}

In this section the complex-based control design proposed in Section II, and their possible implementations discussed in Section IV, are illustrated by an academic example and an application in the field of electrical machines. First, the complex sliding mode control technique is applied to a CVSS. The example is worked out using the control law (12) in Proposition 2, which is more robust than that in Proposition 
1 as it does not require knowledge of the plant's model. Then, the reaching time reduction property of complex-based sliding mode controllers with respect to the standard version is illustrated using the same CVSS. Finally, a rotor flux complexvalued sliding observer for an induction motor is proposed in Section V-C.

\section{A. Nonlinear example}

Consider the nonlinear complex-valued controlled system based on Example 13 in [14], defined by (2) with

$$
f(z)=\left(\begin{array}{c}
z_{1}^{2}-j z_{1}+z_{2} \\
-2 z_{1}-z_{2}
\end{array}\right), \quad g(z)=\left(\begin{array}{c}
2-j \\
j
\end{array}\right) .
$$

As shown in [14], function $f$ is holomorphic and the openloop $(u=0)$ system has two equilibrium points, the origin and $\left(z_{1}^{*}, z_{2}^{*}\right)=(2+j,-4-2 j)$, which are stable and unstable, respectively. The control objective is to stabilise the system at the unstable equilibrium point, namely,

$$
z_{1}^{d}=2+j, \quad z_{2}^{d}=-4-2 j .
$$

Resorting to the error variables $e_{i}=z_{i}-z_{i}^{d}, i=1,2$, (22) becomes

$$
f(e)=\left(\begin{array}{c}
e_{1}^{2}+(4+j) e_{1}+e_{2} \\
-2 e_{1}-e_{2}
\end{array}\right), \quad g(e)=\left(\begin{array}{c}
2-j \\
j
\end{array}\right),
$$

where $e=\left(e_{1}, e_{2}\right)^{T}$.

Defining the complex switching function as

$$
\sigma:=e_{1}
$$

the equivalent control, obtained from (4), results in

$$
u_{e q}=-\frac{2+j}{5}\left[e_{1}^{2}+(4+j) e_{1}+e_{2}\right] .
$$

Proposition 2 guarantees the existence of complex sliding modes using control law (12) whenever (11) is met. It is immediate from (23), (24) that condition (11a) is fulfilled, with

$$
\left|\frac{\partial \sigma}{\partial e} g(e)\right|=\sqrt{5}=\epsilon_{1} .
$$

On the other hand, recalling Remark 3 the control gain, $\kappa \in$ $\mathbb{C} \backslash\{0\}$, is selected as

$$
\kappa=|\kappa| e^{j \varphi_{\kappa}}
$$

and fulfilling (19), i.e.

$$
\varphi_{\kappa}=-\varphi_{\sigma^{\prime} g}(z)=\arctan \left(\frac{1}{2}\right) ;
$$

then, (11b) boils down to

$$
|\kappa|-\left|e_{1}^{2}+(4+j) e_{1}+e_{2}\right|>\epsilon_{2}>0 .
$$

Once sliding motion is guaranteed, the complex-valued ideal sliding dynamics on $\sigma=0$ is given by

$$
\dot{e}_{2}=-\frac{4+2 j}{5} e_{2}
$$

which tends asymtoptically to $e_{2}=0$ because [14]

$$
\operatorname{Re}\left(-\frac{4+2 j}{5}\right)=-\frac{4}{5} \text {. }
$$
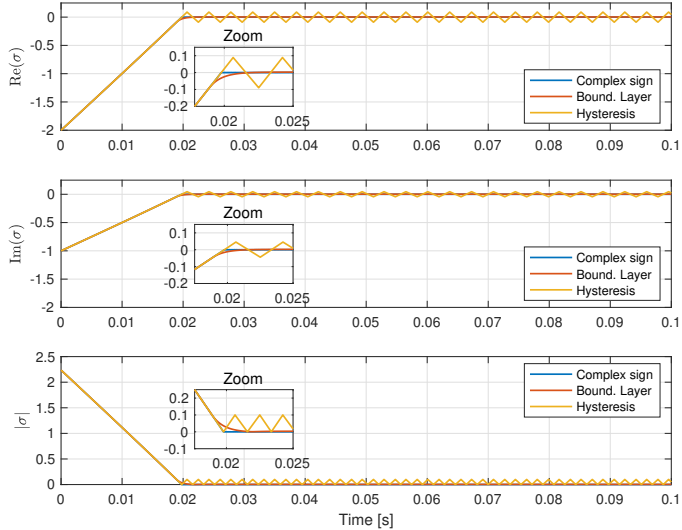

Fig. 2. Real part (top), imaginary part (mid), and modulus (bottom) of the complex switching function, $\sigma(t)$, of system (22), (12) for different implementations: complex sign function (blue), boundary layer approximation (red), and hysteretic approach (yellow).
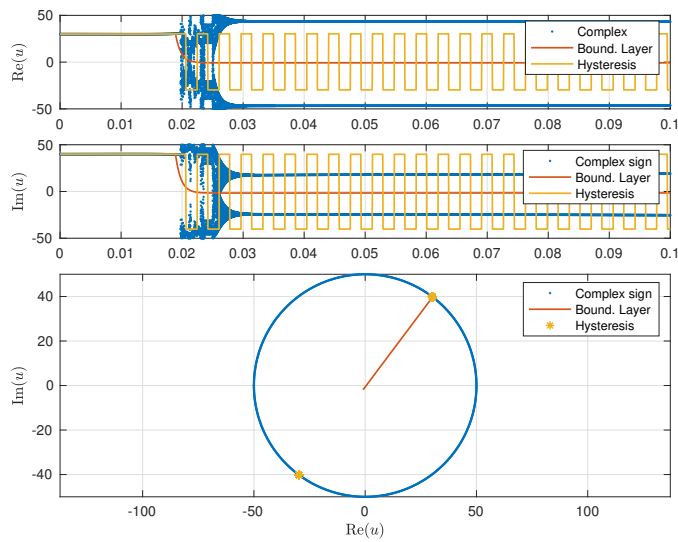

Fig. 3. Real part (top), imaginary part (mid), and complex plane (bottom) plot of the control action, $u(t)$, of system (22), (12) for different implementations: complex sign function (blue), boundary layer approximation (red), and hysteretic approach (yellow).

A numerical simulation has been run in Matlab to test the proposed algorithm. The control gain is set to $|\kappa|=50$, and parameters for the sigmoid and hysteretic approximations are $\epsilon_{b}=\epsilon_{h}=0.1$. The software used a fixed-step solver with $10^{-6} s$ as fundamental sampling time.

Figures 2 and 3 show the complex switching function and the complex control input using the complex sign function (12), the boundary layer solution (21), and the complex hysteresis implementation proposed in Algorithm 1. Notice in Figure 2 that, as the boundary layer approximation becomes continuous in a neighborhood of $\sigma=0$, finite convergence is replaced by an asymptotic behavior with small error. On the other hand, when the hysteretic approximation is used, oscillations around $\operatorname{Re}(\sigma), \operatorname{Im}(\sigma)$ occur, limited by a $|\sigma|<\epsilon_{h}$. Differently from the usual case in multi-input classic sliding modes, $\sigma=0$ is reached simultaneously by the real and imaginary parts of $\sigma$.

The control actions are plotted in Figure 3. Notice that 

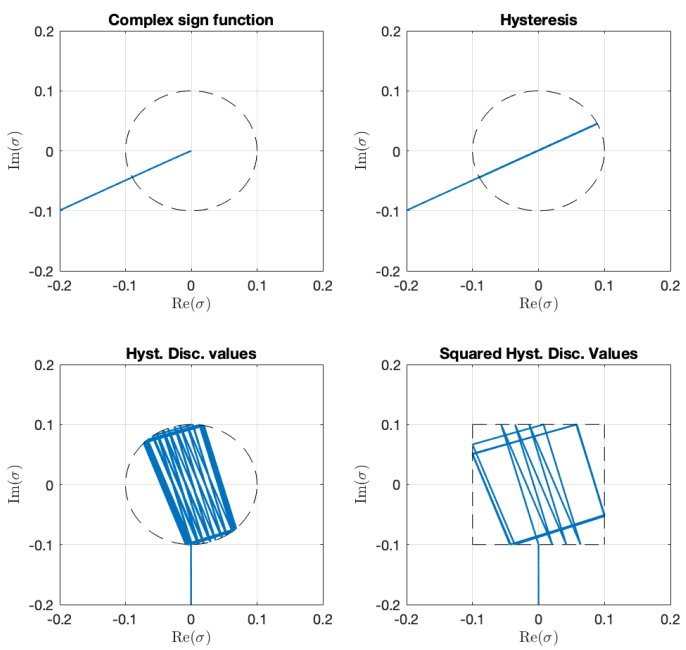

Fig. 4. Complex plane plots of the switching function, $\sigma(t)$, of system (22), (12) for different implementations: complex sign function (top left), hysteretic approximation (top right), hysteretic approximation with four admissible control values (bottom left), squared hysteretic approximation with four admissible control values (bottom right).

the resulting values from (12) and Algorithm 1 are switched and belong to a circle with radius $|\kappa|$ (recall (27)), while the control signal from the boundary layer approximation (21) is continuous and takes values closer to the steady-state equivalent control, namely, $u=0$ according to (25). It is worth clarifying that the control values obtained from the sign function (12) are displayed by dots, and the vertical lines linking them have been omitted: this is to allow including the three signals in the same plot, otherwise this one would hide the other two, and also to show that the switching frequency is that of the time step of the numerical solver. In turn, it is immediate that the control signal (12) values fill the circle with radius $|\kappa|$ during the transient, because in the steady-state it switches between two values. Finally, it has been numerically observed that the transient may be shortened by reducing the sampling time of the simulation.

Figure 4 compares the values of the complex switching function $\sigma$ for different control implementations: the complex sign function (12), the hysteretic approximation in Algorithm 1 , the hysteretic approximation with four admissible control values of Algorithm 3, and the squared hysteretic approximation with four admissible control values of Algorithm 4. It is worth noticing that a limit cycle seems to appear in the hysteretic area when the implementation constrains the admissible control values. Examples of applications where the control values are limited include electrical power drives and converters.

\section{B. Complex versus standard sliding mode control}

CVSS systems admit a real representation by splitting real and imaginary parts, the resulting system dimension being twice the original one. The system described by (22) is equivalent to

$$
\dot{x}=f(x)+g(x) v
$$

with $x \in \mathbb{R}^{4}, v \in \mathbb{R}^{2}$,

$$
f(x)=\left(\begin{array}{c}
x_{1}^{2}+x_{3}+x_{2}-x_{2}^{2} \\
2 x_{1} x_{2}-x_{1}+x_{4} \\
-2 x_{1}-x_{3} \\
-2 x_{2}-x_{4}
\end{array}\right), \quad g(x)=\left(\begin{array}{cc}
2 & 1 \\
-1 & 2 \\
0 & -1 \\
1 & 0
\end{array}\right),
$$

where $z_{1}=x_{1}+j x_{2}, z_{2}=x_{3}+j x_{4}$ and $u=v_{1}+j v_{2}$ have been used. Notice that (29) is now a fourth-order system with two inputs and two outputs, with references set to $x_{1}^{d}=2$ and $x_{2}^{d}=1$ so as to make the problem equivalent to that considered in Section V-A.

A standard sliding mode controller for system (29) is proposed defining

$$
s=\left(\begin{array}{l}
s_{1} \\
s_{2}
\end{array}\right)=\left(\begin{array}{l}
x_{1}-x_{1}^{d} \\
x_{2}-x_{2}^{d}
\end{array}\right),
$$

and using

$$
u_{x}=-k M\left(\begin{array}{l}
\operatorname{sign}\left(s_{1}\right) \\
\operatorname{sign}\left(s_{2}\right)
\end{array}\right),
$$

with $k \in \mathbb{R}^{+}$and

$$
M=\left\|\frac{\partial s}{\partial x} g(x)\right\|\left(\frac{\partial s}{\partial x} g(x)\right)^{-1}=\frac{1}{\sqrt{5}}\left(\begin{array}{cc}
2 & -1 \\
1 & 2
\end{array}\right),
$$

as a control action. Notice that, in accordance with the discussion in Section III, $k M$ is the real analogous of $\kappa_{x}$ in (18), with $M$ standing for $e^{\varphi_{\kappa_{x}}}$ and fulfilling (19): it has a unitary (induced) 2-norm, and the first row corresponds to the complex number with argument $\arctan \frac{1}{2}$ (see also (27)-(28)). Moreover,

$$
\left\|\frac{\partial s}{\partial x} g(x)\right\|=\|\sqrt{5} M\|=\sqrt{5},
$$

as happens in the complex case (26). Therefore, setting

$$
k=\frac{|\kappa|}{\sqrt{2}}=\frac{50}{\sqrt{2}}
$$

the reaching time of this classic design can be fairly compared -in the sense of Section III- with that obtained in Subsection $\mathrm{V}$-A for a complex design.

As predicted in Section III, Figure 5 shows that the reaching time is lower when using the complex approach. Notice also in Figure 6 (top) how in the complex design the target is attained in a straightforward manner, while for the standard design this is attained in a hierarchical manner, i.e. sliding firstly occurs for one component. Finally, Figure 6 (bottom) illustrates the fact that standard sliding mode control only uses four control combinations, while the complex-based controller may take any value on the circumference of radius $|\kappa|$.

\section{A complex-based sliding mode observer}

Rotor flux observation in induction motors is a known problem in the control of electrical drives that attracts attention within the electrical community, see examples in [24] or more recently in [25]. A rotor-flux observer for an induction machine will be designed in this subsection to exemplify the use of complex-valued sliding modes in an engineering application. 

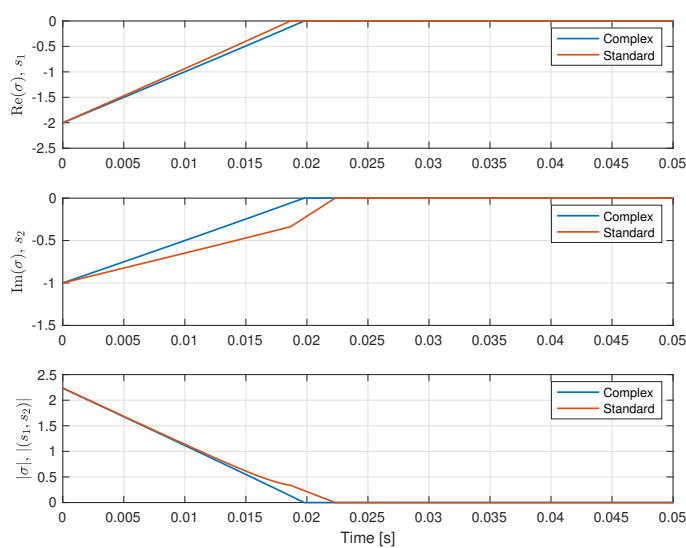

Fig. 5. Complex-based sliding control (blue) versus standard sliding (red) using the complex switching surface function/switching surface vector: real/first component (top), imaginary/second component (mid), modulus/norm (bottom).
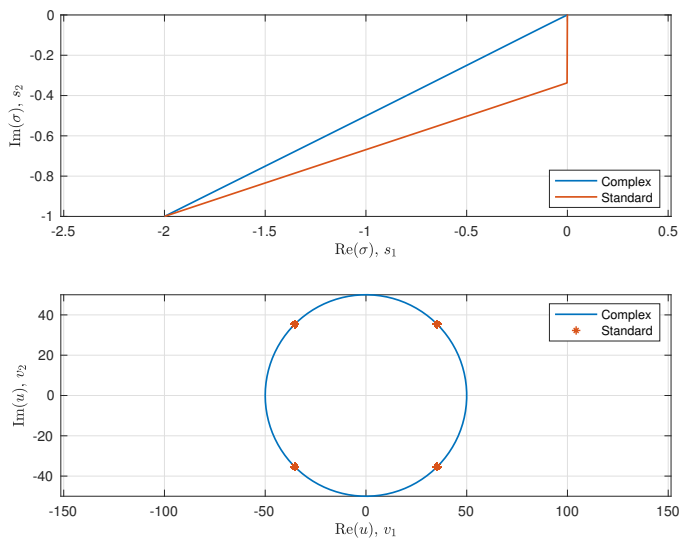

Fig. 6. Complex-based sliding control (blue) versus standard sliding (red) using polar/plane plots of the switching function/switching surface vector (top), and complex control variable/control vector (bottom).

The complex vector representation of the inductor motor is thoroughly developed in [26]. The electrical equations of the inductor motor are:

$$
\begin{aligned}
\frac{\mathrm{d} i_{s}}{\mathrm{~d} t} & =-\gamma i_{s}+\beta\left(\eta-j n_{p} \omega\right) \psi_{r}+\frac{1}{\mu L_{s}} v_{s} \\
\frac{\mathrm{d} \psi_{r}}{\mathrm{~d} t} & =-\left(\eta-j n_{p} \omega\right) \psi_{r}+\eta M i_{s},
\end{aligned}
$$

where $i_{s}, \psi_{r} \in \mathbb{C}$ are the stator current and rotor flux, $\omega \in \mathbb{R}$ is the mechanical speed $v_{s} \in \mathbb{C}$ is the stator voltage, which is used as a control input, $n_{p} \in \mathbb{R}^{+}$is the number of pole pairs,

$$
\mu=\left(1-\frac{M^{2}}{L_{s} L_{r}}\right), \quad \eta=\frac{R_{r}}{L_{r}}
$$

are the leakage factor and rotor time constant, respectively,

$$
\gamma=\frac{R_{s}}{\mu L_{s}}+\frac{R_{r} M^{2}}{\mu L_{s} L_{r}^{2}}, \quad \beta=\frac{M}{\mu L_{s} L_{r}},
$$

$L_{s}, L_{r}, M \in \mathbb{R}^{+}$are the stator, rotor and mutual inductances, and $R_{s}, R_{r} \in \mathbb{R}^{+}$are the stator and rotor resistances modelling the inductor loses.
Assuming that all the parameters are known and the mechanical speed is measurable the proposed observer, similarly to [27], is

$$
\begin{aligned}
\frac{\mathrm{d} \hat{i}_{s}}{\mathrm{~d} t} & =-\gamma \hat{i}_{s}+\beta\left(\eta-j n_{p} \omega\right) \hat{\psi}_{r}+\frac{1}{\mu L_{s}} v_{s}-u \\
\frac{\mathrm{d} \hat{\psi}_{r}}{\mathrm{~d} t} & =-\left(\eta-j n_{p} \omega\right) \hat{\psi}_{r}+\eta M \hat{i}_{s}+l u
\end{aligned}
$$

where $l, u \in \mathbb{C}$. Defining the observation error as

$$
\begin{gathered}
\tilde{i}_{s}=\hat{i}_{s}-i_{s} \\
\tilde{\psi}_{r}=\hat{\psi}_{r}-\psi_{r},
\end{gathered}
$$

the error dynamics becomes

$$
\begin{aligned}
& \dot{\tilde{i}}_{s}=-\gamma \tilde{i}_{s}+\beta\left(\eta-j n_{p} \omega\right) \tilde{\psi}_{r}-u, \\
& \dot{\tilde{\psi}}_{r}=\eta M \tilde{i}_{s}-\left(\eta-j n_{p} \omega\right) \tilde{\psi}_{r}+l u .
\end{aligned}
$$

Proposition 3: Let

$$
\sigma:=\tilde{i}_{s}
$$

be a complex switching function, and let $\mathcal{S}$ denote the corresponding switching manifold. Assume that there exist $\Omega_{1} \subseteq \mathbb{C}^{2}$ with $\Omega_{1} \cap \mathcal{S} \neq \emptyset$, and $k, \epsilon_{2} \in \mathbb{R}^{+}$such that, for all $\left(\tilde{i}_{s}, \tilde{\psi}_{r}\right) \in \Omega_{1}$ :

$$
k-\left|-\gamma \tilde{i}_{s}+\beta\left(\eta-j n_{p} \omega\right) \tilde{\psi}_{r}\right|>\epsilon_{2} .
$$

Then, the switched control action

$$
u=k \operatorname{sign}\left(\tilde{i}_{s}\right)
$$

induces sliding motion of system (31) on $\Omega_{1} \cap \mathcal{S}$. Moreover, for all $\left(\tilde{i}_{s}(0), \tilde{\psi}_{r}(0)\right)$ close enough to $\Omega_{1} \cap \mathcal{S}, \tilde{i}_{s}$ converges to zero in finite time.

Proof: Let $z_{1}=\tilde{i}_{s}, z_{2}=\tilde{\psi}_{r}$. Then, system (31) can be written as (2), with

$$
f(z)=\left(\begin{array}{c}
-\gamma z_{1}+\beta\left(\eta-j n_{p} \omega\right) z_{2} \\
\eta M z_{1}-\left(\eta-j n_{p} \omega\right) z_{2}
\end{array}\right), \quad g(z)=\left(\begin{array}{c}
-1 \\
l
\end{array}\right),
$$

while the switching surface (32) is now:

$$
\sigma:=z_{1} \text {. }
$$

Notice that

$$
\frac{\partial \sigma}{\partial z} g(z)=-1
$$

which, on the one hand, allows to compute the equivalent control following (4), this yielding

$$
u_{e q}=-\gamma z_{1}+\beta\left(\eta-j n_{p} \omega\right) z_{2}
$$

on the other hand, it guarantees the fulfillment of (11a). In turn, taking into account Remark 3, (33) is equivalent to (11b) with $\kappa=-k, k \in \mathbb{R}^{+}$, because $\varphi_{\kappa}$ has been selected as

$$
\varphi_{\kappa}=-\varphi_{\sigma^{\prime} g}=-\pi \text {. }
$$

hence, the result follows straightforward from Proposition 2.

Once on sliding motion, the ideal sliding dynamics follows replacing $\tilde{i}_{s}=0$ and $u=u_{e q}$ in (31b), namely,

$$
\dot{\tilde{\psi}}_{r}=-(1-l \beta)\left(\eta-j n_{p} \omega\right) \tilde{\psi}_{r} .
$$



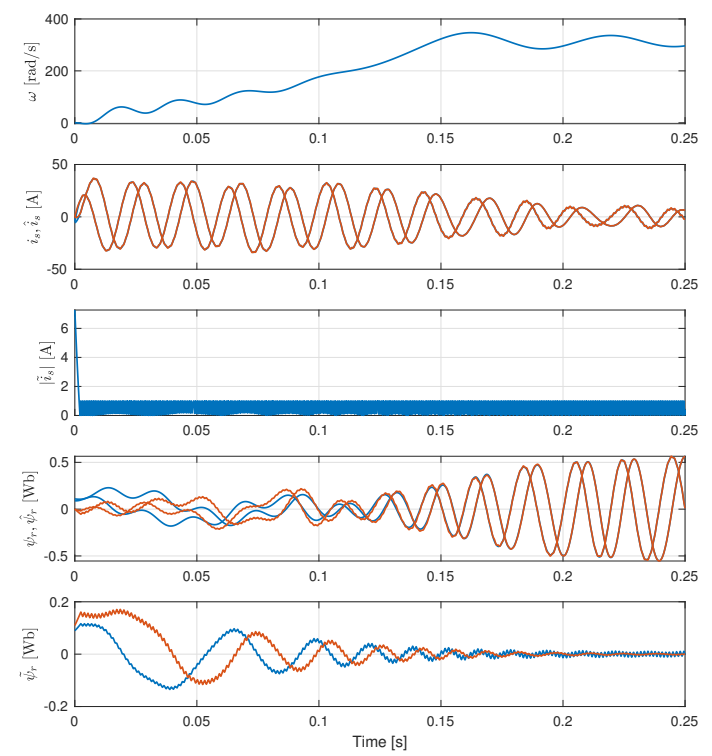

Fig. 7. Rotor-flux observation for an induction motor. From top to bottom: mechanical speed, $\omega$; real an imaginary parts of the stator current (blue) and its observed values (red), $i_{s}, \hat{i}_{s}$; absolute error observation $\left|\tilde{i}_{s}\right|$; real an imaginary parts of the rotor flux (blue) and its observed values (in red), $\psi_{s}, \hat{\psi}_{s}$; real and imaginary components of the observation error, $\tilde{\psi}_{r}$.

Notice that

$\operatorname{Re}\left(-(1-l \beta)\left(\eta-j n_{p} \omega\right)\right)=-(1-\operatorname{Re}(l) \beta) \eta+\operatorname{Im}(l) \beta n_{p} \omega$, and being $\eta, \beta, n_{p}>0$ it results that a selection fulfilling

$$
\operatorname{Re}(l) \eta+\operatorname{Im}(l) n_{p} \omega<\frac{\eta}{\beta}
$$

ensures $\tilde{\psi}_{r} \rightarrow 0$ asymptotically.

Numerical simulation have been run to test the observer proposed in (30). The selected induction motor was a two pole-pairs machine with the following parameters: $L_{s}=$ $L_{r}=109.3 \mathrm{mH}, M=100 \mathrm{mH}, R_{s}=2.7 \Omega, R_{r}=0.5 \Omega$, $J=0.001 \mathrm{~kg} \cdot \mathrm{m}^{2}, b=0.001 \mathrm{~N} \cdot \mathrm{m} \cdot \mathrm{s}$. The observer gains were: $k=50$ and $l=-0.5-0.1 j$, and the control function (34) was implemented using Algorithm 1 with a hysteresis band $\epsilon_{h}=1$. The test consisted in connecting the induction motor to a 200V, $50 \mathrm{~Hz}$ voltage. The obtained results, gathered in Figure 7, show that the observation of the rotor-flux is achieved thanks to the sliding motion induced on the absolute error observation of the stator currents, $\tilde{i}_{s}$, as observed in the mid plot.

\section{CONClusions}

In this paper, sliding modes for complex-valued dynamical systems have been analysed. The proposed methodology results in a generalisation of standard sliding modes with the main advantage of considering more admissible control values, which directly translates into an improvement of the reaching time. Some implementation approximations have also been discussed. The proposal has been numerically validated in an academic example and an electrical application.

\section{REFERENCES}

[1] L. Harnefors, "Modeling of three-phase dynamic systems using complex transfer functions and transfer matrices," IEEE Trans. on Industrial Electronics, vol. 54, no. 4, pp. 2239-2248, 2007.

[2] A. Dòria-Cerezo, M. Bodson, C. Batlle, and R. Ortega, "Study of the stability of a direct stator current controller for a doubly-fed induction machine using the complex Hurwitz test," IEEE Trans. on Control Systems Technology, vol. 21, no. 6, pp. 2323-2331, 2013.

[3] A. Dòria-Cerezo and M. Bodson, "Design of controllers for electrical power systems using a complex root locus method," IEEE Trans. on Industrial Electronics, vol. 63, no. 6, pp. 3706-3716, 2016.

[4] A. Dòria-Cerezo, F. M. Serra, and M. Bodson, "Complex-based controller for a three-phase inverter with an LCL filter connected to unbalanced grids," IEEE Trans. on Power Electronics, vol. 34, no. 4, pp. 3899-3908, 2019.

[5] P. Katz, "Dynamics and control of rotating bodies," IEEE Trans. on Automatic Control, vol. 17, no. 4, pp. 522-526, 1972.

[6] M. J. Hinich and E. Mendes, "Estimating the complex transfer function of a non-linear system," Journal of Sound and Vibration, vol. 259, no. 3 , pp. 715-723, 2003.

[7] A. Hiroshi, Complex-valued neural networks. Theories and applications. World Scientific, 2003.

[8] X. Guo, W. Wu, and Z. Chen, "Multiple-complex coefficient-filter-based phase-locked loop and synchronization technique for three-phase gridinterfaced converters in distributed utility networks," IEEE Trans. on Industrial Electronics, vol. 58, no. 4, pp. 1194-1204, 2011.

[9] E. Frank, "On the zeros polynomials with complex coefficients," Bulletin of the American Mathematical Society, vol. 5, no. 2, pp. 144-157, 1946.

[10] S. D. Agashe, "A new general Routh-like algorithm to determine the number of RHP roots of a real or complex polynomial," IEEE Trans. on Automatic Control, vol. 30, no. 4, pp. 406-409, 1985.

[11] M. Benidir and B. Picinbono, "The extended Routh's table in the complex case," IEEE Trans. on Automatic Control, vol. 36, no. 2, pp. 253-256, 1991

[12] A. Dòria-Cerezo and M. Bodson, "Root locus rules for polynomials with complex coefficients," in Proc. 21st Mediterranean Conference on Control and Automation, 2013.

[13] O. Troeng, B. Bernhardsson, and C. Rivetta, "Complex-coefficient systems in control," in Proc. 2017 American Control Conference, 2017.

[14] T. Fang and J. Sun, "Stability analysis of complex-valued nonlinear differential systems," Journal of Applied Mathematics, p. 7 pages, 2013.

[15] _ _ "Stability analysis of complex-valued nonlinear delay differential systems," Systems \& Control Letters, vol. 62, no. 910-914, 2013.

[16] B. M. Shepit and J. K. Pieper, "Sliding-model control design for a complex valued sliding manifold," IEEE Trans. on Automatic Control, vol. 48, no. 1, pp. 122-127, 2004.

[17] S. Gutman and G. Leitmann, "Stabilizing feedback control for dynamical systems with bounded uncertainty," in Proc. Conference on Decision and Control, 1976.

[18] C. M. Dorling and A. S. I. Zinober, "Two approaches to hyperplane design in multivariable variable structure control systems," International Journal of Control, vol. 44, no. 1, pp. 65-82, 1986.

[19] E. P. Ryan and M. Corless, "Ultimate boundedness and asymptotic stability of a class of uncertain dynamical systems via continuous and discontinuous feedback control," IMA Journal of Mathematical Control \& Information, vol. 1, pp. 223-242, 1984.

[20] V. Utkin, J. Guldner, and J. Shi, Sliding mode control in electromechanical systems. CRC Press, 2009.

[21] G. Bachman and L. Narici, Functional Analysis. NY: Academic Press, INC, 1966.

[22] J. J. Slotine and S. S. Sastry, "Tracking control of nonlinear systems using sliding surfaces, with application to robot manipulators," International Journal of Control, vol. 38, no. 465-492, 1983.

[23] M. J. Corless and G. Leitmann, "Continuous state feedback guaranteeing uniform ultimate boundedness for uncertain dynamic systems," IEEE Trans. on Automatic Control, vol. 26, no. 5, pp. 1139-1144, 1981.

[24] R. Marino, P. Tomei, and C. M. Verrelli, Induction Motor Control Design. Springer, 2010.

[25] A. Pyrkin, A. Bobtsov, A. Vedyakov, R. Ortega, A. Vediakova, and M. Sinetova, "DREM-based adaptive observer for induction motors," in Proc. 58th Conference on Decision and Control, 2019.

[26] D. W. Novotny and T. A. Lipo, Vector Control and Dynamics of AC Drives. London, U.K.: Oxford Univ. Press, 1996.

[27] S. Drakunov and V. I. Utkin, "Sliding mode observers. Tutorial," in Proc. 34th Conference on Decision and Control, 1995. 